\section{The Adenine and Thymine Molecules. Some Excited Singlet and Triplet Levels of the Normal and Tautomeric Forms}

\author{
Julio Marañon and Oscar M. Sorarrain \\ Departamentos de Física y Química, Facultad de Ciencias \\ Exactas, Universidad Nacional de La Plata \\ (Z. Naturforsch. 32 c, 647-649 [1977] ; received \\ December 17, 1976/March 11, 1977) \\ Adenine, Thymine, Barriers, Singlets, Triplets
}

The shape of the barrier for the $\mathrm{H}$ transfer between the normal and tautomeric forms have been determined for the adenine and thymine molecules. The correlations for several singlet and triplet states has been calculated using the CNDO/2-CI method. The relative stability between both conformations and the agreement with some experimental values is also discussed.

\section{Introduction}

The theoretical analysis for the molecules of adenine and thymine has been undertaken by former authors using semi-empirical and ab-initio methods ${ }^{1-4}$. In these papers several results with their biological applications has been reported for the normal and tautomeric conformers in both molecules.

The relative stability for both conformers in each molecule is one of the interesting problems considered by the mentioned authors. In this paper, we have calculated the shape of the barrier between both conformers for a particular movement as another point of view for the theoretical analysis of the stability corresponding to the different molecular conformers.

For this purpose, several excited singlet and triplet levels have been obtained using the semiempirical CNDO/2-CI method.

\section{Method and Results}

In a previous paper ${ }^{5}$ we have discussed the relative stability of the normal and tautomeric conformations in the ground electronic configuration. Now, we have calculated the shape of the barrier corresponding to the proton transfer between both conformers in a group of singly excited singlet and triplet states. We have used a standard CNDO/2-CI program. The molecular parameters (bond angles and interatomic distances) has been taken from the

Requests for reprints should be sent to Dr. O. M. Sorarrain, Facultad de Ciencias Exactas, Departamento de Fisica, Calle 115 y 49 C.C. No. 67, La Plala, Argentina. values given by Sutton ${ }^{6}$ for both molecules. We have taken 30 configurations with a $0.2 \AA$ mash for the movement of the $\mathrm{H}$ atom.

In Fig. 1, we show the relative position of the $\mathrm{H}$ atom in both conformations: normal and tautomeric.

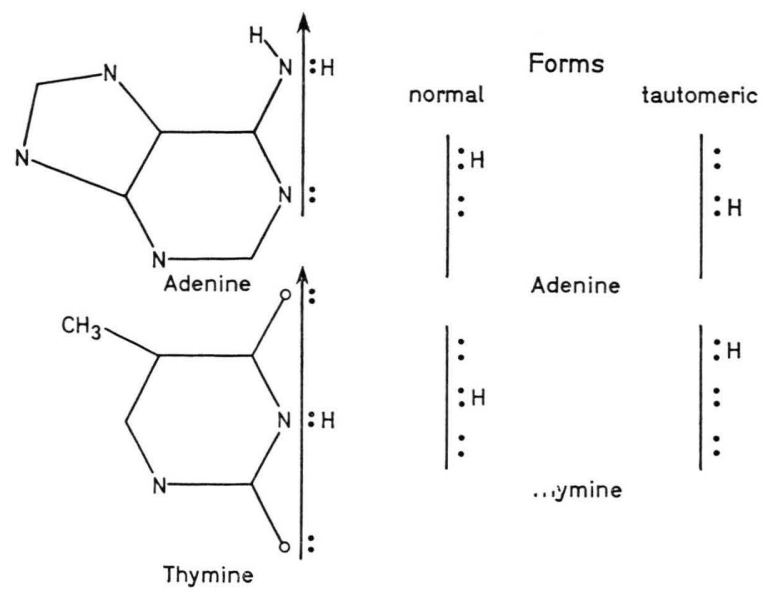

Fig. 1. Normal and tautomeric conformers adenine and thymine.

The excited singlet levels calculated for the molecule of adenine correspond to the first and third spectral region following the classification given by Tinoco ${ }^{1}$. The energy levels for the first spectral range are: $3.92,4.31,4.72$, and $4.95 \mathrm{eV}$. For this range the only available experimental value is $4.9 \mathrm{eV}$. In the third region $(5.9-6.7 \mathrm{eV})$ we have determined the following values: $5.55,5.92,6.12$, 6.44 , and $6.87 \mathrm{eV}$.

The correlation between the excited energy levels corresponding to the different $\mathrm{H}$ positions have been determined following the criterion given by $\mathrm{Anh}^{7}$ (no crossing for levels with the same symmetry). In general, there are difficulties in the case, when is in the middle of the distance between both conformers because there is also a contribution from configurations of both symmetries (even or odd) for some excited levels. Hence these dubious cases we have left out from our figures. The results are shown in Figs $2-5$.

In Fig. 2, we give the singlet $\sigma-\pi^{*}$ and $\pi-\pi^{*}$ transitions for the molecule of adenine.

The values for the $\pi-\pi^{*}$ and $\sigma-\pi^{*}$ triplet transitions are shown in Fig. 3. The excited triplet levels for the normal conformer are: $2.75,3.26$, and $3.52 \mathrm{eV}$. These values obtained in our calculations should be compared with the only one available experimental value: $3.9 \mathrm{eV}^{9}$. 
For the thymine molecule we have calculated some lines for the normal conformer: 4.64, 5.82, 6.42 , and $6.60 \mathrm{eV}$. The agreement with the experimental values 4.67 and $5.94 \mathrm{eV}$ is really satisfactory ${ }^{8}$. The correlation between both conformers for the $\sigma-\pi^{*}$ and $\pi-\pi^{*}$ excited singlet and triplet states are given in Figs 4 and 5.

Summing up: the values given in Figs $2-5$ show the following: a) The tautomeric conformer of the molecule of adenine is more stable for some singlet and triplet states (see Figs $2-3$ ), i.e.: the normal conformation has low stability for some singlet $\pi-\pi^{*}$ levels. On the contrary, the $\sigma-\pi^{*}$ singlets corresponding to the normal conformer are more stable than the tautomeric ones (there is one exception, the 1st excited level, see Fig. 2).

b) In the case of the two conformers of the thymine molecule we have similar troubles than the

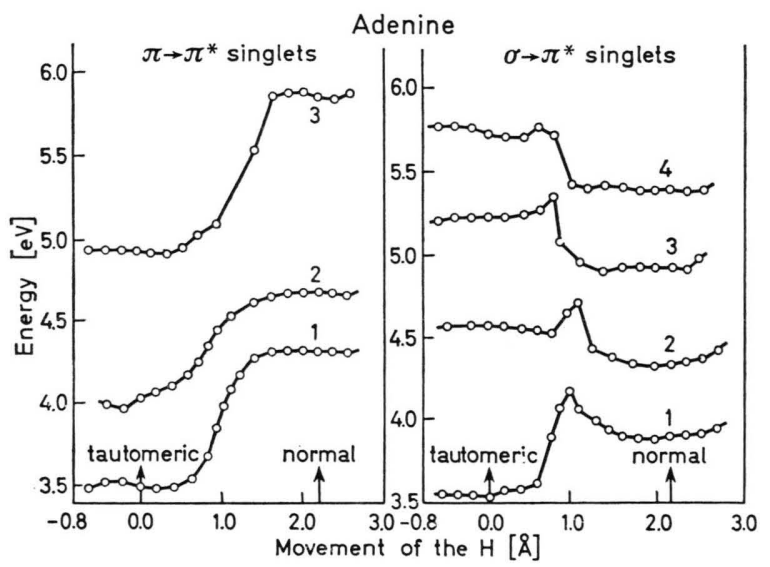

Fig. 2. Shape of the barrier between both conformers. Excited singlet levels, molecule of adenine.

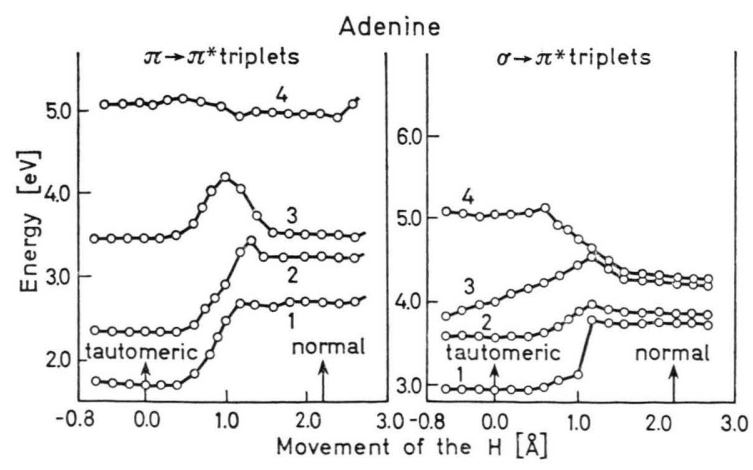

Fig. 3. Shape of the barrier between both conformers. Excited triplet levels, molecule of adenine. former authors ${ }^{3,4}$. Anyway, some results are interesting enough to be pointed out, i.e.: the lst $\sigma-\pi^{*}$ singlet transition predicts a more stable conformation of the normal molecule and the $\sigma-\pi^{*}$ triplet one of the tautomeric form.

\section{Final Comments}

We have determined the intersection of the energy surface (two coordinates are necessary for the movement of the $\mathrm{H}$ in the molecular plane) with a plane normal to the movement of the $\mathrm{H}$ in the molecular plane. The results often show that the tautomeric forms are more stable than the normal ones both the adenine and thymine molecules. If these conclusions are right it should be possible to determine experimentally the tautomeric equilibrium constants not only of the electronic ground state ${ }^{\mathbf{1 0}}$, but also for some excited levels of the free nucleotide.

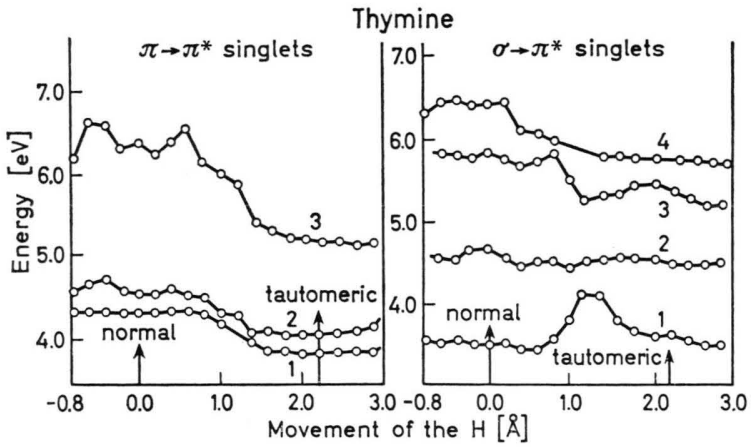

Fig. 4. Shape of the barrier between both conformers. Excited singlet levels, molecule of thymine.

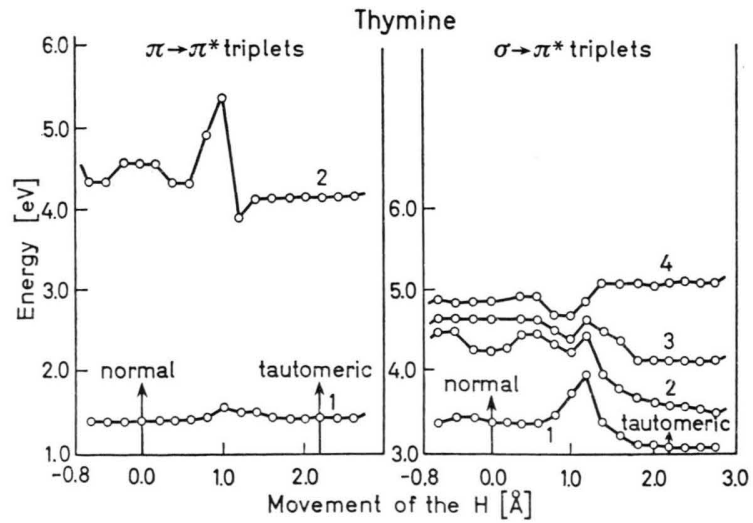

Fig. 5. Shape of the barrier between both conformers. Excited triplet levels, molecule of thymine. 
If the bases are within the DNA molecule a radiative excitation process should lead to the tautomeric forms: $A^{*}, T^{*}$. These conformers have another pairing pattern than normal one: $A^{*}-C$ and $\mathrm{G}-\mathrm{T}^{*}$. These errors can propagate at cell duplication following the schema given by Löwdin ${ }^{11}$. A single excitation could cause thus changes in the

1 A. Pullman and B. Pullman, Advances in Quantum Chemistry, Vol. 4, 267, Academic Press, New York-London 1968.

2 H. Fujita, A. I. Imamura, and C. Nagata, Bull. Chem. Soc. Japan 43, 1467 [1969].

3 M. Tanaka and S. Nagakura, Theoret. Chim. Acta (Berl.) 6, 320 [1966].

${ }^{4}$ H. Morita and S. Nagakura, Theort. Chim. Acta (Berl.) 11, 279 [1968].

5 O. M. Sorarrain and E. A. Castro, Chem. Phys. Letters 19, 422 [1973].

${ }^{6}$ L. E. Sutton, Tables of Interatomic Distances and Configuration in Molecules and Ions, P. M. 181, The Chemical Society, London 1965. genetic information, but the whole process is very complicated because the rate of decay from the excited level to the ground state must also be considered.

We should like to express our gratitude to the Consejo Nacional de Investigaciones de Argentina and the O. E. A. for their help during the development of this paper.

${ }^{7}$ H. Trong Anh, Las reglas de Woodward-Hoffmann, Editorial Reverté, Barcelona 1974.

8 J. Ladik and K. Appel, Theoret. Chim. Acta (Berl.) 4, 132 [1965].

9 A. Pullman, E. Kochanski, M. Gilbert, and A. Denis, Theoret. Chim. Acta (Berl.) 10, 231 [1968].

10 A. R. Katritzky and A. J. Waring, J. Chem. Soc. 1962, 1540 .

11 P. O. Lowdin, Quantum Genetics and the Aperiodic Solid, Preprint B7, Quantum Chemistry Group, Uppsala University 1963. 